\title{
Efecto de dos cepas de Trichoderma en el control de Botrytis cinerea y la calidad del fruto en fresa (Fragaria sp.)
}

\section{Effect of two Trichoderma strains on Botrytis cinerea control and fruit quality for the strawberry (Fragaria sp.)}

JULIA BIBIANA MERCHÁN-GAITÁN'

ROSA LILIA FERRUCHO²

JAVIER GIOVANNI ÁLVAREZ-HERRERA ${ }^{3,4}$

Planta de fresa var. Ventana.

Foto: J.B. Merchán-Gaitán

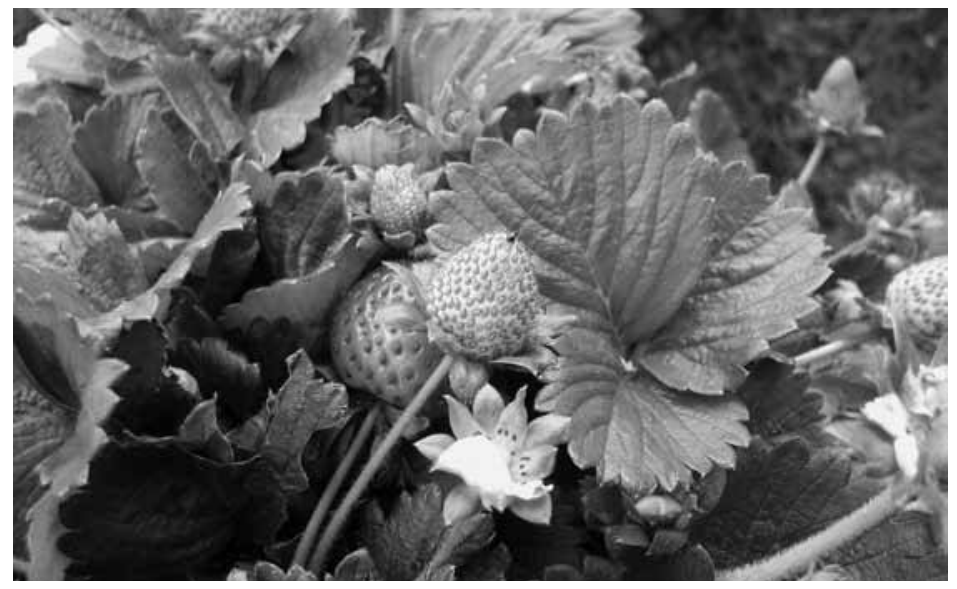

\section{RESUMEN}

El moho gris en fresa causado por Botrytis cinerea es una enfermedad limitante ya que disminuye la calidad y el valor comercial de los frutos. El manejo de esta enfermedad se basa en el empleo de fungicidas químicos aplicados al fruto. Por esta razón, el uso de organismos biocontroladores es una alternativa para el control de esta patología ya que permite disminuir los residuos de pesticidas en el ambiente. Se evaluaron dos cultivares comerciales de fresa (Camino Real y Ventana), en un diseño completamente al azar con 4 tratamientos, Trichoderma harzianum y T. lignorum, iprodione en dosis comercial y un tratamiento testigo, cada uno con tres repeticiones, para un total de doce unidades experimentales (UE), cada UE compuesta por cinco plantas. La incidencia de la enfermedad en el tratamiento testigo fue del 60\%, mientras que para los tratamientos con $T$. harzianum y T. lignorum solo alcanzó un 33\%, lo que indica un control mayor de los antagonistas en comparación con el químico sobre la enfermedad. En cuanto a la severidad, los tratamientos con T. harzianum y T. lignorum redujeron significativamente en un $32 \%$ el desarrollo del patógeno en la planta. La masa fresca de frutos mostró diferencias significativas cuando se aplicó T. lignorum, ya que este tratamiento obtuvo los frutos más grandes y con mayor tonalidad roja. La aplicación de Trichoderma sp. presentó frutos con mayor firmeza en las dos variedades, con valores promedio de $9,1 \mathrm{~N}$, lo que indica que estos frutos soportan mejor la manipulación poscosecha y el almacenamiento.

\footnotetext{
Escuela de Posgrado, Programa de Maestría en Ciencias Agrarias, Universidad Nacional Agraria La Molina, Lima (Perú).

2 Laboratorio Nacional de Diagnóstico Fitosanitario, Instituto Colombiano Agropecuario (ICA), Tibaitatá, Mosquera (Colombia).

3 Facultad de Ciencias Agropecuarias, Grupo de Investigaciones Agrícolas, Universidad Pedagógica y Tecnológica de Colombia, Tunja (Colombia).

4 Autor para correspondencia. jgalvarezh@gmail.com
} 


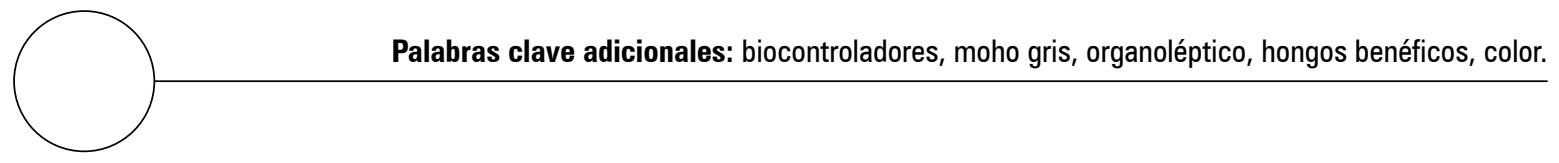

\section{ABSTRACT}

The gray mold caused by Botrytis cinerea is a limiting disease in strawberry and decreases the quality and commercial value of the fruit. Management of this disease is based on the use of chemical fungicides applied to the fruits. For this reason, the use of biocontrol organisms is an alternative for the control of this disease as they help to reduce pesticide residues in the environment. Two commercial cultivars of strawberry (Camino Real and Ventana) were evaluated in a completely randomized design with four treatments: Trichoderma harzianum, T. lignorum, iprodione in commercial doses and a control treatment, each one with three replicates for a total of twelve experimental units (EU), with each EU comprising five plants. The incidence of the disease in the control treatment was $60 \%$; whereas, the T. harzianum and T. lignorum treatments only reached $33 \%$, indicating greater control of the antagonists as compared with the chemical treatment of the disease. Regarding severity, the treatments with $T$. harzianum and $T$. lignorum significantly reduced the development of the pathogen in the plant by $32 \%$. The fresh mass of the fruits presented significant differences when $T$. lignorum was applied; this treatment obtained the heaviest fruits and more red color (hue). The application of Trichoderma sp. provided firmer fruits in the two varieties with average values of $9.1 \mathrm{~N}$. This indicates that these fruits will probably support better post-harvest handling and storage.

Additional key words: biocontrol, gray mold, organoleptic, beneficial fungi, color.

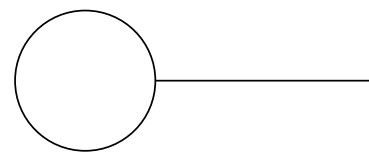

INTRODUCCIÓN

La fresa es un cultivo de gran valor económico para los productores agrícolas de los departamentos en Cundinamarca y Boyacá (Colombia). Además, los frutos son muy apetecidos por las características nutricionales y organolépticas; sin embargo son altamente perecederos y susceptibles al ataque de patógenos durante la cosecha y en la poscosecha por lo que disminuyen rápidamente su calidad comercial (Ruiz y Piedrahíta, 2012). Las pérdidas poscosecha se deben principalmente al ataque del hongo Botrytis cinerea, el cual genera daños en los frutos de fresa tales como: pérdida de firmeza, color y sabor, que conducen a una disminución en la vida útil (Thompson, 2003). Por lo anterior, los frutos requieren de aplicaciones de fungicidas de síntesis química en precosecha y poscosecha para prolongar su vida en anaquel (Esmel et al., 2004) lo que contribuye a incrementar los costos de producción.

Los estudios sobre pérdidas en poscosecha realizados en los mercados de Nueva York y Chicago, demuestran que estas pueden llegar a ser de 28,8\% y $41,2 \%$, respectivamente, por daños mecánicos y pudriciones causadas por hongos (Kader, 1991). Así mismo, el moho gris es considerado como la enfermedad más importante de la fresa, el cual puede llegar a generar pérdidas de hasta el $50 \%$ de la producción (Averre et al., 2003). El hongo Botrytis cinerea se manifiesta inicialmente como una mancha marrón clara o amarillenta hacia el final del cáliz y a los pocos días cubre de un moho gris, 
de apariencia polvosa, toda la superficie de la fruta (Agrios, 2005). Este es capaz de afectar el 95\% de los frutos después de 48 horas de cosechados (Chaves y Wang, 2004).

Consecuentemente, los agricultores siempre han intentado minimizar las pérdidas causadas por plagas y enfermedades con la aplicación de métodos aparentemente efectivos, los cuales pueden ser nocivos tanto para el ambiente como para el hombre al consumir los productos tratados (Castaño, 2002). Debido al desarrollo y producción de plaguicidas de síntesis química, los productores de fresa como primera medida disponen de estos, gracias a su fácil adquisición, para realizar el control de patógenos, por lo menos a corto plazo (Castaño, 2002). Sin embargo, estos productos encierran riesgos, los cuales se hacen evidentes cuanto más prolongado e intensivo es su uso (López, 2007).

Existen varios fungicidas recomendados para prevenir $B$. cinerea, entre los cuales están los bencimidazoles, dicarboximidas, anilinopirimidinas y carboxamidas (Russel, 2004). Sin embargo, la aplicación de estos productos tiene restricciones debido al periodo de carencia y la residualidad (Zapata et al., 2002). En la actualidad, a nivel comercial se encuentra disponible para el control de $B$. cinerea un agente de control biológico que tiene como principio activo el hongo antagonista T. harzianum (Harman et al., 1996), además de este existen otros biocontroladores como Gliocladium spp., y también algunas bacterias como el Bacillus subtilis que pueden tener potencial de control de esta enfermedad en fresa (Abbott Laboratories de Chile Ltda., 1997). Al respecto, diferentes especies del genero Trichoderma han sido reconocidas por sus características como biocontrolador de patógenos del suelo y bacterias (Mishra et al., 2014), además de ser habitantes naturales del mismo (Kim y Knudsen, 2013).

En estudios de control de enfermedades con $T$. harzianum se ha observado que este hongo no solo reduce la severidad sino que también induce la estimulación del crecimiento de las plantas
(Lynch et al., 1991; Cubillos et al., 2009), del mismo modo, se ha demostrado el efecto de T. lignorum en el control de Rhizoctonia solani en plantas de haba (Aziz et al., 1997). Este efecto también es reportado en experimentos con plantas libres de patógenos bajo condiciones controladas (Mishra et al., 2014). T. harzianum, viride y lignorum han mostrado diferentes mecanismos de acción biocontroladora sobre hongos fitopatógenos, ya que estos compiten por espacio y nutrientes, micoparasitan y realizan antibiosis, lo cual tiene una acción directa sobre el hongo patógeno (Infante et al., 2009). Así mismo, T. harzianum mejora la producción de frutos en cuanto a masa fresca y calidad (Harman et al., 2004).

Por lo anterior, el objetivo de esta investigación fue evaluar la aplicación de dos cepas de Trichoderma, T. harzianum y T. lignorum en el control del moho gris causado por Botrytis cinerea en dos variedades de fresa (Camino Real y Ventana) y la incidencia de estos controladores en la calidad de los frutos.

\section{MATERIALES Y MÉTODOS}

El estudio se realizó en Tunja en un área bajo cubierta plástica, la cual se encuentra ubicada a $73^{\circ} 23^{\prime}$ de longitud oeste y $5^{\circ} 32^{\prime}$ de latitud norte a una altura de $2.782 \mathrm{msnm}$, con una humedad relativa del $70 \%$ y una temperatura promedio de $18^{\circ} \mathrm{C}$ dentro de la cubierta.

La siembra de estolones de fresa previamente desinfectados con Benomil ${ }^{\circledR}$ se realizó en bolsas plásticas negras con capacidad de $3 \mathrm{~kg}$. El suelo que se utilizó fue solarizado y desinfectado con medios físicos (agua caliente) siguiendo la metodología de Schreiner et al. (2001). Las plántulas de las variedades Ventana y Camino Real fueron colectadas de un cultivo que presentaba buenas condiciones fitosanitarias, el cual estaba sembrado a una altura de $1 \mathrm{~m}$ del suelo. Al mes de ser trasplantados los estolones, se realizó la aplicación de fertilizantes de crecimiento (40-3-5); al 
tercer mes se aplicó fertilizante de floración (1530-15). El riego se realizó cada 3 d.

Para la inoculación, se aisló $B$. cinerea de frutos de fresa enfermos obteniendo 10 aislamientos en cajas de Petri. Se preparó una suspensión de conídias en $20 \mathrm{~mL}$ de agua destilada a una concentración de $3,2 \times 10^{5}$ conídias/mL. El recuento se realizó en una cámara de Neubauer (Celeromics, Valencia, España). La inoculación se hizo con una fumigadora manual, asperjando las plántulas un mes después de ser trasplantadas.

El diseño experimental que se utilizó fue completamente al azar con cuatro tratamientos correspondientes a T1: testigo con inóculo del patógeno; T2: control químico comercial Rovral ${ }^{\circledR}$ (fungicida cuyo ingrediente activo es Iprodione); T3: control biológico comercial Tricho $\mathrm{D}^{\circledR}$ (ingrediente activo T. harzianum); T4: control biológico comercial My$\operatorname{cobac}^{\circledR}$ (ingrediente activo de T. lignorum), para las variedades de fresa evaluadas. Cada tratamiento tuvo tres repeticiones, para un total de 12 unidades experimentales (UE), cada una compuesta por cinco plantas de las dos variedades. Los tratamientos se aplicaron en forma de aspersión.

Se realizaron dos aplicaciones en cada tratamiento a intervalos de 1 mes con una fumigadora manual Garden (Royal Cóndor, Soacha, Colombia) de capacidad de 1,8 L que posee una boquilla ajustable con dosis de pulverización de $150 \mathrm{~mL} \mathrm{~min}{ }^{-1}$. Los tratamientos se aplicaron de la siguiente manera: (i) el fungicida Rovral en dosis de $1,5 \mathrm{~mL} \mathrm{~L}^{-1}$ de agua, (ii) el producto comercial Tricho $\mathrm{D}^{\circledR}$ en concentración de 1 x $10^{8}$ de conídias/g en una suspensión de $5 \mathrm{~g} \mathrm{~L}^{-1}$ de agua y (iii) Mycobac ${ }^{\circledR}$ en concentración de $2 \times 10^{7}$ de conidias/g en una suspensión de $25 \mathrm{~g} \mathrm{~L}^{-1}$ de agua, lo anterior de acuerdo con la ficha técnica de cada producto.

Se realizó el seguimiento a la aparición de síntomas como marchitamiento, pérdida de turgencia, presencia de micelio y pudrición, durante el desarrollo de la planta, en especial en flores y frutos. Se efectuó un muestreo al final del expe- rimento el cual tuvo una duración de 4 meses, en el que en las dos últimas cosechas, se tomaron cinco frutos al azar por planta, para un total de 25 frutos por unidad experimental de cada variedad y se determinó la incidencia de la pudrición causada por B. cinerea. Además se calculó el índice de severidad en las plantas y se evaluaron algunos parámetros de calidad del fruto.

El índice de severidad (IS) se calculó con los datos de severidad de la enfermedad obtenidos mediante escala descriptiva (tabla 1) a partir de la ecuación 1 y siguiendo la metodología empleada por Orjeda (1998).

$I S=\frac{0 * N_{o}+5 * N_{1}+10 * N_{2}+25 * N_{3}+50 * N_{4}+100 * N_{5}}{N T}$

Donde $\left(N_{\alpha} N_{1} N_{2^{\prime}} N_{3^{\prime}} N_{4} N_{5}\right)$ corresponde al número de plantas incluidas en cada categoría y NT es el número total de plantas evaluadas.

En el fruto, se determinó la masa fresca medida con una balanza electrónica Acculab VIC 612 de 0,01 g de precisión (Sartorius Group, Göttingen, Alemania), los sólidos solubles totales con un refractómetro HANNA HI 96803 (Hanna Instruments, Eibar, España). El pH se determinó con un potenciómetro HANNA HI 8424 (Hanna Instruments, Eibar, España). El color mediante un colorímetro PCE-RGB (PCE-Ibérica, Albacete, España) y se utilizó el índice color CIRG empleado por Carreño et al. (1997) (ecuación 2). El diámetro se midió con un calibrador Mitutoyo

Tabla 1. Índice de severidad por categorías

\begin{tabular}{|l|l|}
\hline Categoría & Síntomas en la planta (\%) \\
\hline 0 & Sanas \\
\hline 1 & Menos del 5 \\
\hline 2 & 6 al 10 \\
\hline 3 & 11 al 25 \\
\hline 4 & 26 al 50 \\
\hline 5 & Más del 50 \\
\hline
\end{tabular}


Digimattic CD6"CSX y la firmeza con un penetrómetro PCE-PTR200 (PCE-Ibérica, Albacete, España).

$C I R G=\left(\frac{(180-H)}{(L+S)}\right)$

Donde: CIRG (color index of red grapes) es el índice de color, (H) es la intensidad de color, (L) luminosidad del color y $(\mathrm{S})$ es la saturación del color.

Se calculó la eficiencia económica que consistió en dividir la masa fresca de frutos de cada tratamiento dividido en el costo por tratamiento, con el fin de determinar la cantidad de masa comercial producida por cada peso (\$) invertido.

Con los datos obtenidos se realizó una prueba de normalidad, y con el fin de establecer la existencia de diferencias significativas se hicieron análisis de varianza (Anova) para cada una de las variedades de fresa y se efectuó la prueba de Duncan $(P \leq 0,05)$. Para el análisis se utilizó el paquete estadístico SAS v. 8e.

\section{RESULTADOS Y DISCUSIÓN}

\section{Incidencia}

Para esta variable, la prueba de normalidad arrojó que los datos no presentaron un comportamiento normal, por lo tanto se realizó un Anova no paramétrico, en el cual se encontraron diferencias significativas $(P \leq 0,05)$ en la variable incidencia de la enfermedad para los tratamientos. El testigo obtuvo mayor valor de incidencia que los tratamientos en los que se aplicaron los biocontroladores, lo que resalta la importancia de aplicar estos productos empleados en la reducción de la presencia de la enfermedad (figura 1).

Se encontró mayor incidencia de $B$. cinerea en el testigo con un $60 \%$, seguido del tratamiento con la aplicación de Iprodione con un 46,66\% de plantas enfermas, lo que indica una efectividad moderada de este último en el control de la enfermedad. En los tratamientos con Trichoderma se presentó un mayor control de la enfermedad, ya que la $B$. cinerea mostró una incidencia de $33 \%$.

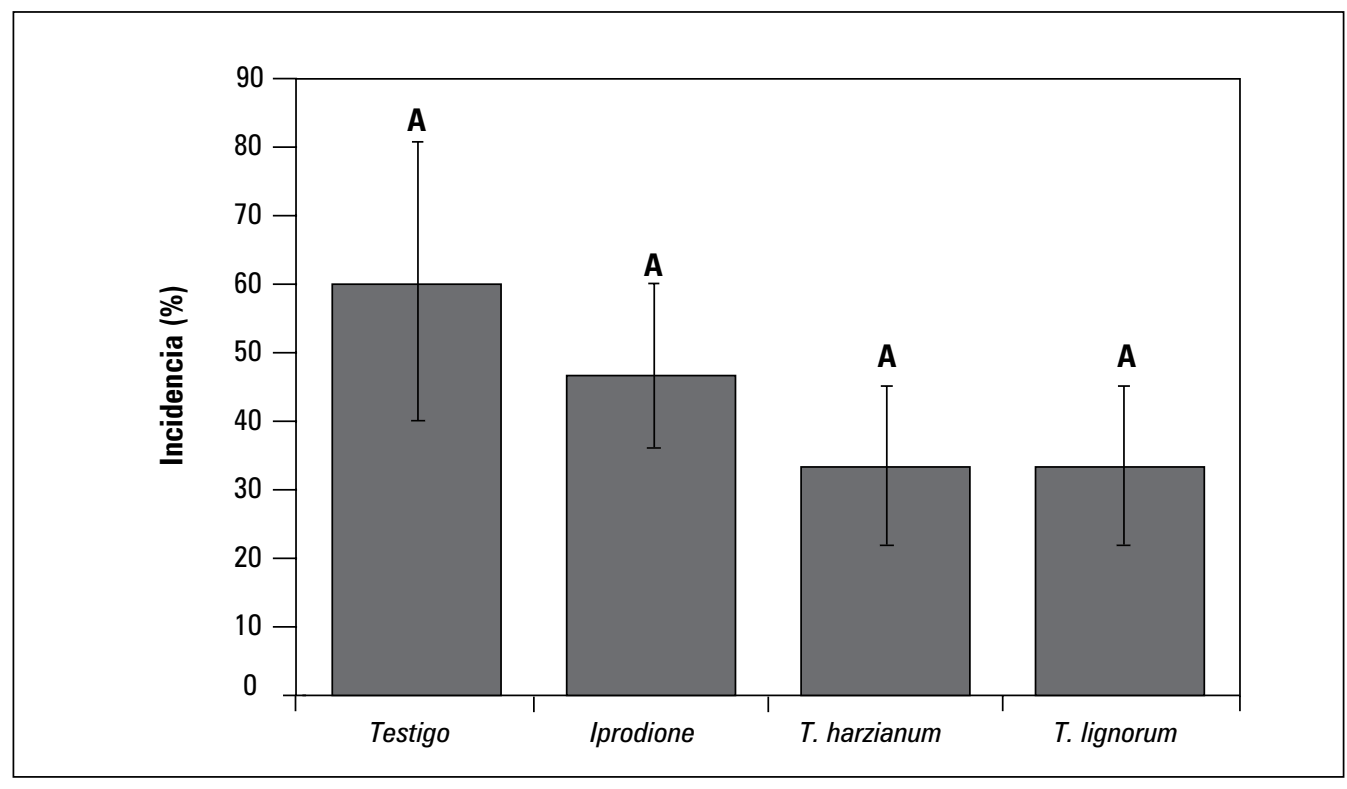

Figura 1. Incidencia de la enfermedad $B$. cinerea en dos variedades de fresa (Camino Real y Ventana) bajo diferentes tratamientos control. Promedios con letras distintas indican diferencia significativa según la prueba de Duncan $(P \leq 0,05)(n=6)$. Barras de error indican el error estándar. 
Es probable que el control del tratamiento con Iprodione tenga un menor efecto, pues su presencia en el suelo está limitada por procesos como la lixiviación (PLM, 2009). El Iprodione tiene un tiempo de vida media de $42 \mathrm{~d}$ en el suelo, mientras que el efecto del Trichoderma es progresivo, pues a medida que se introduce en el suelo, este comienza a propagarse y tiene una rápida colonización (Harman et al., 2004), de esta forma, reduce la población de fitopatógenos, y a la vez, genera una asociación con las raíces de la planta, lo cual favorece la absorción de nutrientes, el crecimiento foliar y la inducción de defensas en la planta (Hohmann et al. 2011). Por tal razón, se puede inferir que se requiere de más aplicaciones de Iprodione que de Trichoderma, lo cual es acorde a lo reportado por Harman et al. (2004).

\section{Índice de severidad}

Se observó que los productos comerciales evaluados de Trichoderma produjeron un control estadísticamente significativo $(P \leq 0,05)$ respecto a la reducción de la $B$. cinerea. Los resultados obtenidos en la prueba de Duncan presentaron un promedio de índice de reducción en la severidad de 3\% para el tratamiento testigo y para el tratamiento con aplicación de Iprodione en las dos variedades, como lo muestra la figura 2. Así mismo, cabe resaltar que los resultados de severidad arrojaron un mejor control de la enfermedad cuando se aplicó T. harzianum y T. lignorum, pues estos presentaron un índice del 18\% de reducción en la severidad de la enfermedad, lo cual pone de manifiesto los beneficios de la aplicación de Trichoderma como controlador de fitopatógenos.

Otros estudios con $T$. harzianum y $T$. lignorum presentaron un control similar de $B$. cinerea inhibiendo su crecimiento en fresa, esto debido a la reducción en la colonización y esporulación del patógeno por competencia del biocontrolador acorde a lo mencionado por Gordon et al. (2000). Otra ventaja de la aplicación de Trichoderma sp., es que este hongo benéfico puede sobrevivir en medios con contenidos altos de pesticidas y otros químicos, ya que extrae nutrientes de los hongos que degrada y ayuda a la descomposición de estas

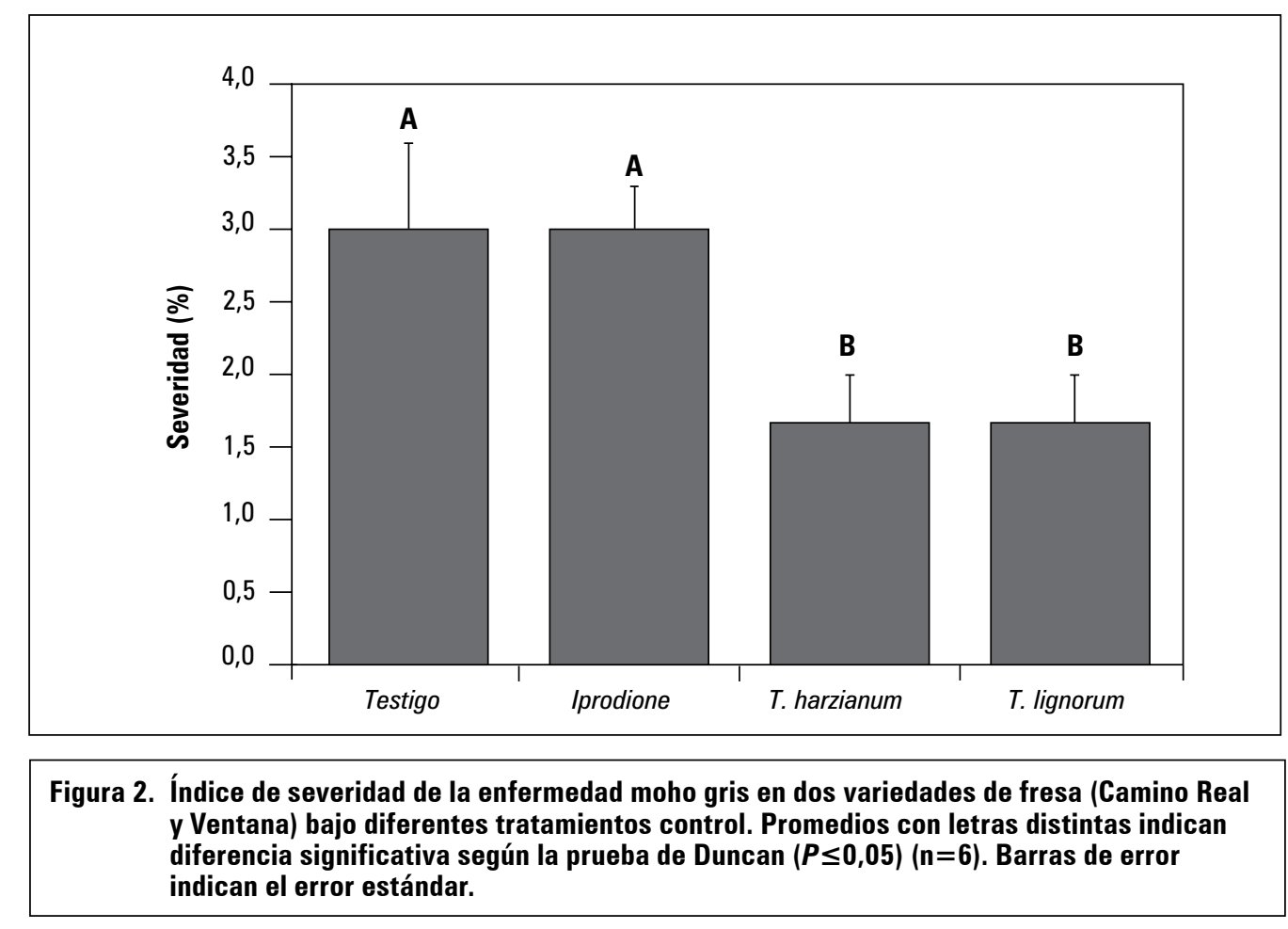


sustancias, con lo cual ve favorecido su efecto al ir acompañado de la adición de materia orgánica y materiales compostados (Ahmad et al., 1987).

El tratamiento testigo mostró en algunas plantas infección por Botrytis, la cual se caracterizó por la formación de micelios atacando el fruto maduro, no obstante, en los tratamientos con aplicación de Trichoderma, el control ejercido por T. harzianum ocurrió probablemente debido al rápido crecimiento que este hongo tiene en el sustrato y a la capacidad para reducir el desarrollo de otros hongos en diferentes órganos de la planta (Guédez et al., 2009).

Se observó una acción como biocontrolador de T. harzianum sobre Botrytis, ya que este antagonista cubrió el hongo que produce la enfermedad y no permitió el esparcimiento hacia otras partes de la planta, similar a lo reportado por Guédez et al. (2009), también es probable que el control se realice debido a que las hifas de Trichoderma sp. se adhieren a las del hospedante mediante la formación de estructuras parecidas a ganchos y apresorios, las cuales se enrollan al- rededor de estas, hecho que limita el crecimiento de estos hongos. Este evento es mediado por procesos enzimáticos posteriores a la fase de reconocimiento (Infante et al., 2009).

Del mismo modo, se pudo apreciar una mayor pérdida de flores en el tratamiento que recibió la aplicación de Iprodione (figura 3), siendo más susceptible la variedad 'Ventana' en los tratamientos testigo e Iprodione, mientras que en los tratamientos con T. harzianum y T. lignorum fue la variedad Camino Real la que presentó menor pérdida floral. Probablemente, una de las razones para el aborto floral es la presencia de Botrytis, la cual pudo haber atacado el pedúnculo o la estructura floral, privándola de nutrientes y agua, generando así la perdida (Sequi, 2004).

\section{Masa fresca de frutos}

La masa fresca de los frutos mostró diferencias significativas $(P \leq 0,05)$ con un promedio de 7,6 $\mathrm{g}$ dando como resultado frutos más grandes con el tratamiento de T. lignorum (figura 4) para las dos variedades de fresa evaluadas, por lo que

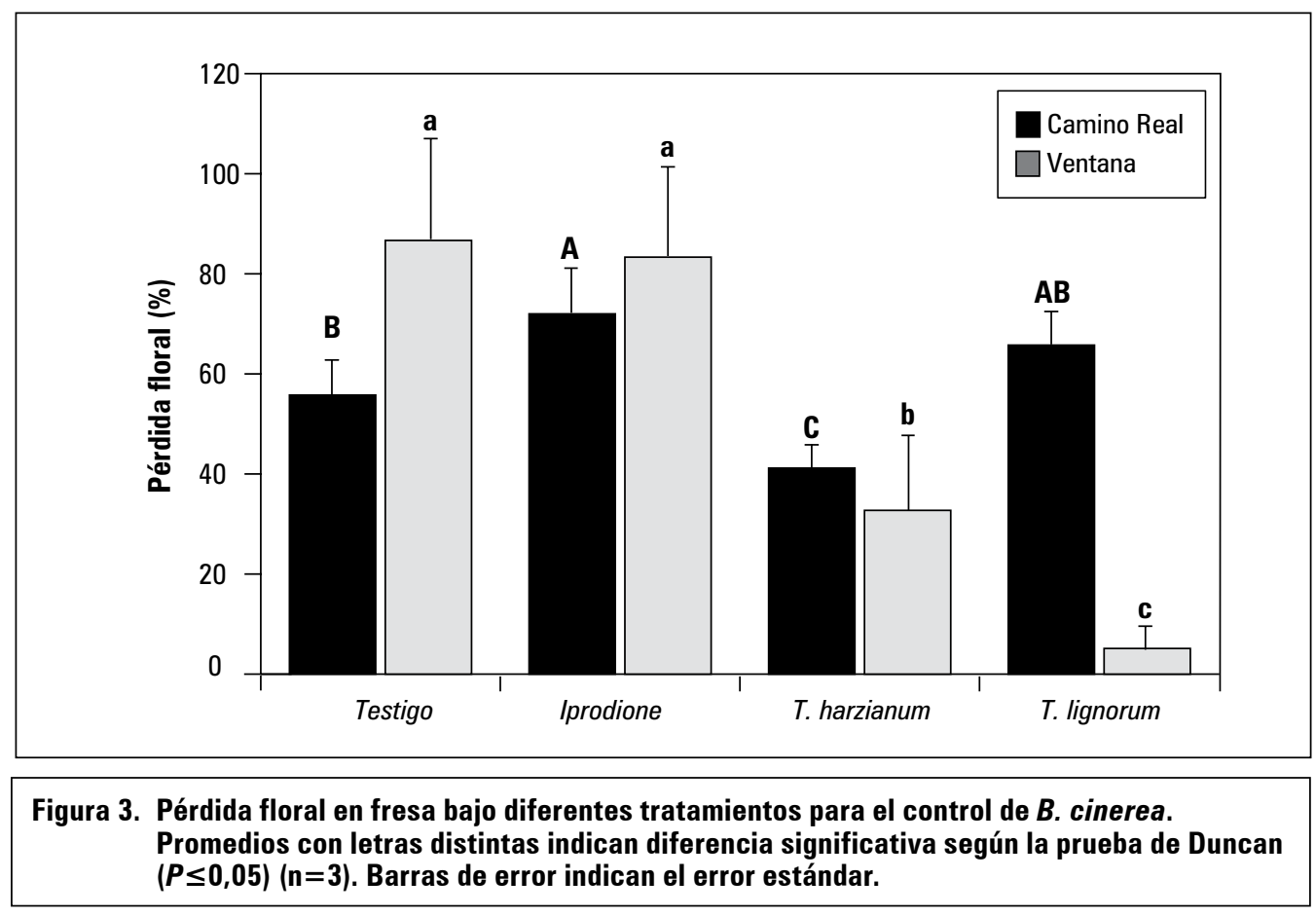




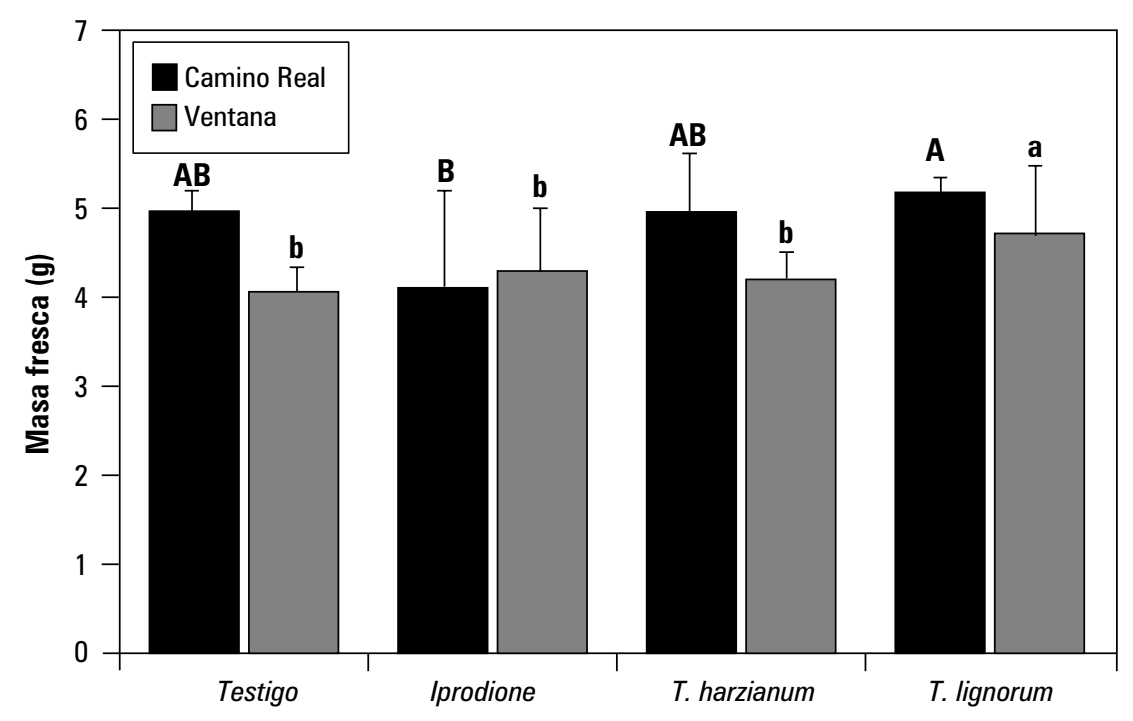

Figura 4. Promedio de la masa fresca del fruto de fresa por variedad bajo diferentes tratamientos para el control sobre el patógeno $B$. cinerea. Promedios de la misma serie con letras distintas indican diferencia significativa según la prueba de Duncan $(P \leq 0,05)(n=3)$. Barras de error indican el error estándar.

se obtuvieron mayores rendimientos agrícolas, calidad biológica del fruto y mayor eficiencia económica, lo que se podría explicar debido al efecto que produce Trichoderma en el crecimiento radicular, el aumento de la disponibilidad de nutrientes y la inducción de defensas (Harman et al., 2004).

Además de los efectos benéficos que producen los biocontroladores sobre los patógenos de la planta, algunas de las especies de Trichoderma son capaces de colonizar las superficies de la raíz y causar cambios sustanciales en el metabolismo de la planta, aumentando la absorción de los nutrientes por parte de la planta y a su vez el crecimiento de la misma, lo que mejora la producción de frutos en cuanto a masa y calidad (Harman et al., 2004). Al respecto, resultados similares fueron encontrados por Jiménez et al. (2011) en tomate, ya que al aplicar T. harzianum en semillero, trasplante y $15 \mathrm{~d}$ después del trasplante obtuvieron mayor crecimiento de la parte aérea y de las raíces, así mismo, Trichoderma tiene un efecto importante en la promoción del crecimiento de las plantas (Mishra et al., 2014).

\section{Tamaño (diámetro ecuatorial)}

El diámetro del fruto no presentó diferencias significativas $(P \leq 0,05)$ en la variedad Ventana mientras que en la variedad Camino Real el Anova mostró diferencias estadísticas (resultados no mostrados), lo cual ocurrió probablemente debido al efecto benéfico que propician las cepas de Trichoderma en la asimilación de nutrientes a la planta (Mishra et al., 2014), aspecto que probablemente redunda en el aumento del área foliar fotosintética que, a su vez, generó frutos de mayor tamaño (Harman et al., 2004). Además, se debe tener en cuenta que el fruto de la variedad Ventana es alargado, mientras que el fruto de Camino Real es globoso (Proplantas, 2011), por lo que es probable que sea más fácil encontrar diferencias significativas en esta última.

\section{Sólidos solubles totales (SST), acidez total titulable (ATT) y $\mathrm{pH}$}

Los SST no presentaron diferencias estadísticas $(P \leq 0,05)$ en ninguno de las variedades (resulta- 
dos no mostrados). Los valores medios mostrados fueron de $4,99 \%$ para el tratamiento con aplicación de $T$. lignorum, valores que resultan bajos comparados con los ${ }^{\circ}$ Brix para fresas de calidad comercial, los cuales se encuentran alrededor del 8\% (Baraona y Sancho, 1998). En cuanto a la ATT, esta no presentó diferencias significativas $(P \leq 0,05)$ para los tratamientos aplicados en ninguna de las variedades. Los promedios de ATT estuvieron alrededor de $0,45 \%$, los cuales son bajos al compararse con los valores reportados por Guédez et al. (2009).

Lo anterior se debe probablemente a que el contenido nutritivo y los SST del fruto cambian según la variedad, volumen del fruto, fertilidad del suelo y condiciones climáticas (Baraona y Sancho, 1998). Aún así, la calidad del fruto no se vio afectada por la aplicación del tratamiento químico ni de Trichoderma, lo que implica que esta última no altera las características de sabor del fruto y tampoco genera residualidad ni periodo de carencia en el fruto, lo que lo hace apto para el consumo.

Los resultados para los valores de $\mathrm{pH}$ no mostraron diferencias significativas $(P \leq 0,05)$, esto indica que ninguno de los tratamientos altera la calidad organoléptica del fruto. Los valores de $\mathrm{pH}$ en promedio fueron de 3,61. Estos valores son similares a los reportados por Aday et al. (2011) en la variedad Camarrosa los cuales están alrededor de 3,63.

\section{Firmeza}

El Anova no mostró diferencias significativas $(P \leq 0,05)$ en cuanto a la resistencia a la penetración del fruto (resultados no mostrados). La firmeza de los frutos de fresa presentó valores promedio para todos los tratamientos y variedades de 9,1 N. Este parámetro de calidad, a menudo se relaciona con la ruptura celular, la pérdida de adherencia celular y la hidrólisis del almidón en azucares por amilasas y fosforilasas (Agravante et al., 1990). Los valores presentados de firmeza son altos al compararse con los obtenidos por Martínez-Bolaños et al. (2008) para el cultivar Festival con promedios de 1,46 N. Lo anterior podría implicar que las variedades $\mathrm{Ca}$ mino Real y Ventana son menos susceptibles al ablandamiento de los frutos causado por $B$. cinerea. Al respecto, está comprobado que $B$. cinerea en presencia de luz incrementa la emisión de etileno en vid (Zhu et al. 2012), lo que a su vez activaría procesos de degradación de pared celular, disminuyendo la firmeza, por lo cual la aplicación de Trichoderma sp. adquiere mayor relevancia para la conservación de la calidad de los frutos de fresa.

\section{Color}

El color del epicarpio para el valor de $\mathrm{R}$ (red) presentó diferencias significativas $(P \leq 0,05)$ con un promedio de 48,4 mientras que en los casos de $\mathrm{G}$ (green) y B (blue) no mostraron diferencias significativas $(P \leq 0,05)$ y los frutos de fresa de las dos variedades presentaron valores de color de 22,3 y 18,6 respectivamente (resultados no mostrados).

Esto indica que hay mayor presencia del color rojo (R), lo que se debe probablemente a que las antocianinas son los principales componentes del fruto de fresa, estas expresadas en el color rojo (Bodelon et al. 2010). Además se midió la intensidad del color $(\mathrm{H})$ con un valor de 7,04, la intensidad del color (S) con valores promedio de 111 y la saturación de color (L) con 30,4, con estos datos se halló el índice del color (CIRG), el cual arrojó diferencias significativas para el tratamiento con la aplicación de T. harzianum con un promedio de 1,5, mientras que entre variedades, la de mayor CIRG fue Ventana con un promedio de 1,8. Es importante la expresión del color rojo, ya que $B$. cinerea al causar ablandamiento en los frutos causa una oxidación en los tejidos que hace que el color del fruto cambie a tonos marrones, con lo cual disminuye la calidad para la comercialización del producto (Kader, 1991). 
A pesar de que las principales normas de calidad para exportación de fresa NTC 4103 mencionan que la coloración de los frutos debe ser homogénea dependiendo del estado de madurez definido en la tabla de colores, esta no especifica el grado de saturación o brillo requerido para establecer las diferentes calidades de fresa para su consumo en fresco. Al respecto, es importante resaltar que una de las características más decisiva para los consumidores es el color externo del fruto (Del-Valle et al. 2005).

\section{Correlación de las variables}

Esta arrojó como resultado que la incidencia y el índice de severidad son directamente proporcionales (figura 5), lo cual indica que al haber un aumento de la incidencia de la enfermedad también se verá afectado el índice de severidad.

Según la grafica de correlaciones se observó una relación inversamente proporcional entre las variables de incidencia e índice de severi- dad con la firmeza, ${ }^{\circ}$ Brix, pH y masa fresca, esto probablemente debido a que el aumento de la incidencia y el índice de severidad de la enfermedad afectan la calidad del fruto lo que disminuye dichas propiedades; sin embargo, se podría inferir que la incidencia de la enfermedad en la planta implicaría el deterioro en la calidad de los frutos. En cuanto al comportamiento de las variables de tamaño (diámetro ecuatorial) y color no muestran relación con los demás parámetros medidos, esto indica que no se vieron afectadas por la aplicación de los tratamientos.

\section{Eficiencia económica}

Al evaluar los costos de cada tratamiento, se pudo observar mayor eficiencia en masa fresca por peso (\$) invertido en el tratamiento testigo, el cual mostró una relación de 0,8 y 0,4 para las variedades Camino Real y Ventana, respectivamente (figura 6), sin embargo la calidad del fruto no fue la mejor.

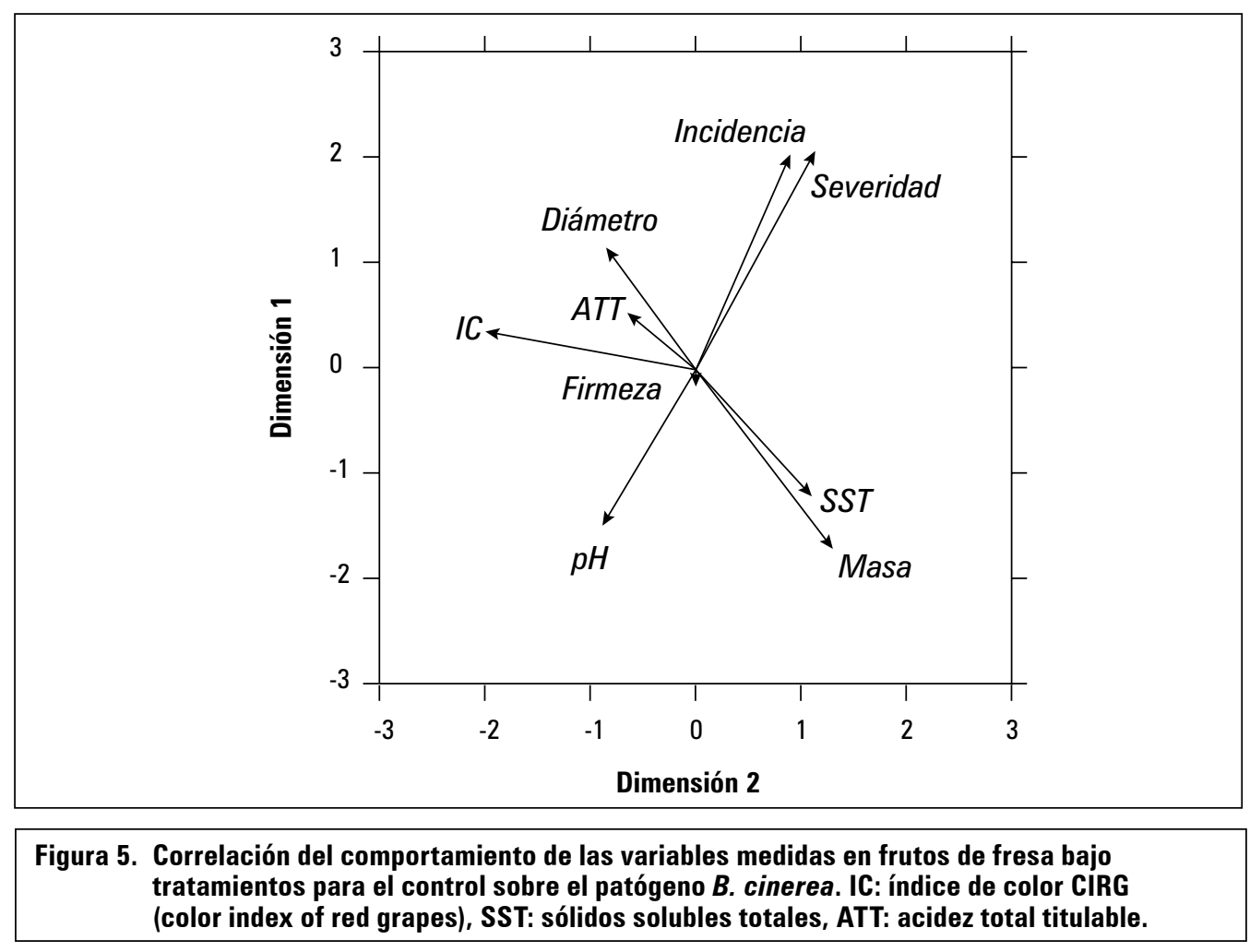

Vol. 8 - No. 1 - 2014 


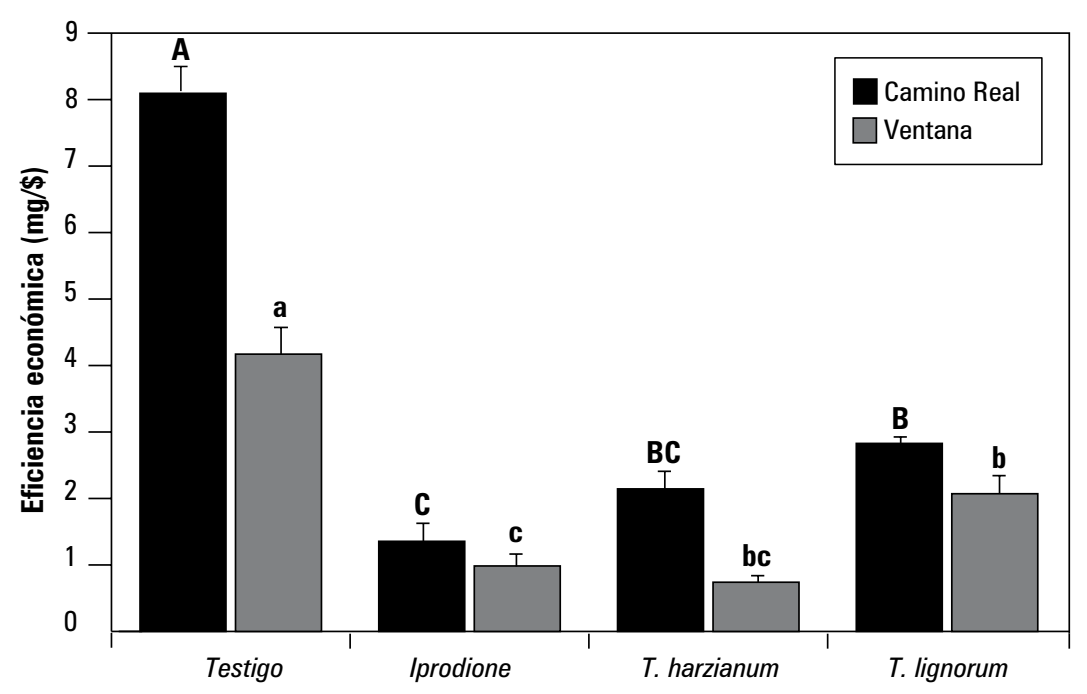

Figura 6. Eficiencia económica de frutos de fresa de dos variedades en relación con el costo de producción, bajo diferentes tratamientos control sobre el patógeno $B$. cinerea. Letras distintas indican diferencia significativa según la prueba de Duncan $(P \leq 0,05)$. Barras de error indican el error estándar.

En cuanto a la calidad de los frutos fue el tratamiento de T. lignorum en las dos variedades el que presentó la mejor eficiencia económica al compararse con los tratamientos de Iprodione y $T$. harzianum, lo cual demuestra que se puede disminuir el impacto del daño por Botrytis y producir frutos de buena calidad a bajo costo.

\section{CONCLUSIONES}

Con T. harzianum y T. lignorum se obtuvo una protección superior a la que brinda el Iprodione en el manejo de $B$. cinerea en fresa, no obstan- te, se presentó un gran porcentaje de pérdida de flores, la cual pudo deberse a variables externas como la deficiencia de boro ya que no hubo aplicación de este elemento. En las variables de calidad de los frutos de fresa, estos presentaron incrementos significativos en la masa y en el color, lo que aumenta la calidad de la fresa. Al aumentar el índice de severidad se afectó la calidad del fruto. La aplicación de T. lignorum es más económica que la de T. harzianum para el control $B$. cinerea en fresa, por lo cual se recomienda la aplicación del primero, ya que este hongo benéfico propició la obtención de frutos de buena calidad.

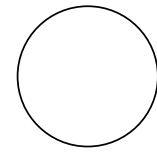

Abbott Laboratories de Chile Ltda. 1997. La alternativa biológica. Área de desarrollo en Botrytis, nuevas estrategias de control cultural, biológico y químico en uva de mesa. Facultad de Ciencias Agrarias, Universidad de Chile, Santiago. pp. 82-88.

Aday, M.S. C. Caner y F. Rahval. 2011. Effect of oxygen and carbon dioxide absorbers on strawberry quality. Postharv. Biol. Technol. 62, 179-187.

\section{REFERENCIAS BIBLIOGRÁFICAS}

Agravante, J.U., T. Matsui y H. Kitagawa. 1990. Starch breakdown and changes in amylase activity during ripening of ethyle and ethano-treated bananas. Acta Hort. 269, 133-140.

Agrios, G. 2005. Plant pathology. $5^{\mathrm{a}}$ ed. Academic Press, New York, NY.

Ahmad, J.S. y R. Baker. 1987. Rhizosphere competence of Trichoderma harzianum. Phytopathol. 77, 182-189. 
Averre, Ch., R. Jones y R. Milholland. 2003. Strawberry diseases and their control. Fruit disease information. Note 5. Plant pathology extension. North Carolina State University, Raleigh, NC.

Aziz, N.H., M.Z. El-Fouly, A.A. EL-Essawy y A.A. Khalaf. 1997. Influence of bean seedling root exudates on the rhizosphere colonization by Trichoderma lignorum for the control of Rhizoctonia solani. Bot. Bull. Acad. Sin. 38, 33-39.

Baraona, M. y E. Sancho. 1998. Manzana, melocotón, fresa y mora. pp. 97-108. En: Fruticultura Especial II. Universidad Estatal a Distancia. San José, Costa Rica.

Bodelon, O.G., M. Blanch, M.T. Sánchez-Ballesta, M.I Escribano y C. Merodio. 2010. The effects of high $\mathrm{CO}_{2}$ levels on anthocyanin composition, antioxidant activity and soluble sugar content of strawberries stored at low non-freezing temperature. Food Chem. 122, 673-678.

Carreño, J., A. Martínez, L. Almela y J.A. Fernández-López. 1997. Chemotaxonomical classification of red table grapes based on anthocyanin profile and external colour. Lebens. Wissen. Technol. 30, 259. 265.

Castaño, Z.J. 2002. Principios básicos de fitoepidemiología. Universidad de Caldas, Manizales, Colombia.

Chaves, N. y A. Wang. 2004. Combate del moho gris (Botrytis cinerea) de la fresa mediante Gliocladium roseum. Agron. Costar. 28(2), 73-85.

Cubillos-Hinojosa, J.N. Valero y L. Mejía. 2009. Trichoderma harzianum como promotor del crecimiento vegetal del maracuyá (Passiflora edulis var. flavicarpa Degener). Agron. Colomb. 27(1), 81-86.

Del Valle, V.P., M.A. Hernández y M.J. Guarda. 2005. Development of cactus mucilage edible coating (Opuntia ficus-indica) and its application to extend strawberry (Fragaria ananassa) shelf life. Food Chem. 91(4), 751-756.

Esmel, C. E., J.R. Duvaly A.A Steven. 2004. The influence of calcium thiosulfate on yieldand postharvest quality of 'Sweet Charlie' strawberry. Proc. Fla. State Hort. Soc. 117, 48-51.

Gordon, L., A. Stensvand y A. Tronsmo. 2000. Efecto de la temperatura y el estrés de nutrientes sobre la capacidad de productos comerciales de Trichoderma para el control de Botrytis cinerea y Mucor piramidal de fresa bajo invernadero. Biol. Control 19, 149-160.

Guédez, C., I. Cañizález, C. Castillo y R. Olivar. 2009. Efecto antagónico de Trichoderma harzianum sobre algunos hongos patógenos postcosecha de la fresa (Fragaria spp.). Rev. Soc. Ven. Microbiol. 29, 34-38.

Harman, G.E., B. Latorre, E. Agosin, R. San Martin, D.G. Riegel, P.A. Nielsen, A. Tronsmo y R.C. Pearson. 1996. Biological and integrated control of Botrytis bunch rot of grape using Trichoderma spp. Biol. Control 7, 259-266.

Harman, G. E., C.R. Howell, A. Viterbo, I. Chet y M. Lorito. 2004. Trichoderma species-opportunistic, avirulent plant symbionts. Nat. Rev. Microbiol. 2, 43-56.

Hohmann, P., E. Jones, R. Hill y A. Stewart. 2011. Understanding Trichoderma in the root system of $\mathrm{Pi}$ nus radiata: associations between rhizosphere colonisation and growth promotion for comercially grown seedlings. Fungal Biol. 115, 759-767.

Infante, D., B. Martínez, N. González y Y. Reyes. 2009. Mecanismos de acción de Trichoderma frente a hongos fitopatógenos. Rev. Protección Veg. 24(1), 14-21.

Jiménez, C., N. Sanabria de Albarracin, G. Altuna y M, Alcano. 2011. Efecto de Trichoderma harzianum (Rifai) sobre el crecimiento de plantas de tomate (Lycopersicon esculentum L.). Rev. Fac. Agron. (LUZ) 28, $1-10$

Kader, A.A. 1991. Quality and its maintenance in relation to the postharvest physiology of strawberry. pp. 145-152. En: Dale, A. y J.J. Luby (eds.). The strawberry into the $21^{\text {st }}$. Timber Press, Portland, OR.

Kim, T.G. y G.R. Knudsen. 2013. Relationship betwen the biocontrol fungus Trichoderma harzianum and the phytopahogenic fungis Fusarium solani f. sp. pisi. Appl. Soil Ecol. 68, 57-60.

López, C.E. 2007. Fitopatología molecular. Universidad Nacional de Colombia, Bogotá.

Lynch J., K. Wilson, M. Ousley y J. Whipps. 1991. Response of lettuce to Trichoderma treatment. Lett. Appl. Microbiol. 12, 56-61.

Martínez-Bolaños, M., D. Nieto-Ángel, D. TelizOrtiz, J. Rodríguez-Alcázar, M.T. Martínez-Damián, H. Vaquera-Huerta y O. Carrilli-Mendoza. 2008. Comparación cualitativa de fresas (Fragaria $x$ ananassa Duch) de cultivares Mexicanos y Estadounidenses. Rev. Chapingo Serie Hort. 14(2), 113-119.

Mishra, A., M. Kumari, S. Pandey, V. Chaudhry, K.C. Gupta y C.S. Nautiyal. 2014. Biocatalytic and antimicrobial activities of gold nanoparticles synthesized by Trichoderma sp. Bioresource Technol. 166, 235-242. 
Orjeda, G. 1998. Evaluación de la resistencia de los bananos a las enfermedades de Sigatoka negra y marchitamiento por Fusarium. Guías técnicas INIBAP 3. IPGRI, Roma, Italia; Red Internacional para el mejoramiento del banano y el plátano, Montpellier, Francia.

PLM. 2009. Diccionario de especialidades agroquímicas. $19^{a}$ ed. Ediciones PLM, México.

Proplantas. 2011. Camino Real. En: www.proplantas. com/files/camino-real.pdf; consulta: marzo de 2014.

Ruiz, R. y W. Piedrahita. 2012. Fresa. pp. 474-495. En: Fischer, G. (ed.). Manual para el cultivo de frutales en el trópico. Produmedios, Bogotá.

Russel, P. 2004 Sensitivity baselines in fungicide resistance research and management. Crop Life International, Bruselas.
Schreiner, P., K. Ivors y J. Pinkerton. 2001. Soil solarization reduces arbuscular mycorrhizal fungi as a consequence of weed suppression. Mycorrhiza 11, 273-277.

Sequi, P. 2004. Los microelementos en la nutrición vegetal. Valagro SpA, Lanciano (Ch), Italia.

Thompson, A. 2003. Almacenamiento en atmósferas controladas de frutas y Hortalizas. Acribia, Zaragoza, España.

Zapata, J.L., A. Saldarriaga, M. Londoño y C. Díaz. 2002. Manejo del cultivo de la uchuva en Colombia. Boletín Técnico 14. Centro de Investigación La Selva, Corpoica, Rionegro, Colombia.

Zhu, P., L. Xu, C. Zhang, H. Toyoda y S. Gan. 2012. Ethylene produced by Botrytis cinerea can affect early fungal development and can be used as a marker for infection during storage of grapes. Postharv. Biol. Technol. 66, 23-29 